\title{
NEUROMUSCULAR TIMING MODIFICATION IN RESPONSES TO INCREASED SPEED AND PROPORTIONAL RESISTANCE WHILE PUSHING A SLED IN YOUNG ADULTS
}

\author{
Martin G. Rosario \\ School of Physical Therapy, Texas Woman's University, Dallas, Texas, United States of America.
}

\begin{abstract}
Introduction: The XPO Sled Trainer used in this research is a novel device that provides low rolling resistance at low speeds with an instant and automatic equivalent increment in resistance with increased speed. Purpose: To examine the impact of using the XPO Trainer on neuromuscular activation time at low and high speeds in young, seemingly healthy adults. Materials and Methods:

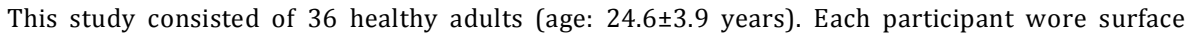
electromyography (EMG) electrodes on their dominant leg over the anterior tibialis and gastrocnemius (GA). The four tasks examined in this work were, walk, run, walk pushing (WP) and run pushing (RP). Participants were asked to walk on their self-selected speed. To execute the tasks, participants walked and ran a distance of 40 feet both with and without the XPO Trainer sled. Subjects completed a total of 3 trials per task (total of 12) with one-minute breaks between tasks to reduce fatigue. Results: Neuromuscular time for activation was significantly modified while pushing the sled in both self-selected speeds, particularly in GA muscle. WP and RP exhibited an inverted relationship in neuromuscular timing patterns. Conclusion/Clinical Relevance: We suggest using the sled while walking to provoke endurance and running to work on strengthening of lower leg musculature. The sled proved to be a useful adjunct tool for neuromuscular training in different alterations of the lower limb muscles, especially the extensor musculature.
\end{abstract}

Keywords: resistance sled, neuromuscular modifications, EMG, muscle activation

\section{MODIFICACIÓN DEL TIEMPO DE RESPUESTA NEUROMUSCULAR EN ADULTOS JÓVENES AL AUMENTO DE LA VELOCIDAD Y LA RESISTENCIA DURANTE EL EMPUJE DE UN TRINEO}

\begin{abstract}
RESUMEN
Introducción: el trineo XPO Trainer utilizado en esta investigación, es un dispositivo novedoso que proporciona baja resistencia a la rodadura a bajas velocidades, con un incremento equivalente instantáneo y automático de resistencia al incrementar la velocidad. Objetivo: Examinar el impacto del uso del XPO Trainer en el tiempo de activación neuromuscular a bajas y altas velocidades en adultos jóvenes y aparentemente sanos. Material y métodos: En este estudio participaron 36 adultos sanos (edad: 24,6 $\pm 3,9$ años). Cada participante llevaba electrodos de electromiografía de superficie (EMG) en su pierna dominante, sobre el tibial anterior y el gastrocnemio (GA). Las cuatro tareas examinadas en este trabajo fueron: caminar, correr, caminar empujando el trineo(WP) y correr empujando el trineo (RP). Se pidió a los participantes que caminaran una velocidad autoseleccionada. Para ejecutar las tareas, los participantes caminaron y corrieron una distancia de 40 pies $(12,2 \mathrm{~m})$ con y sin el trineo XPO Trainer. Los sujetos completaron un total de 3 intentos por tarea (un total de 12), con descansos de un minuto entre tareas para reducir la fatiga. Resultados: el tiempo de activación neuromuscular se modificó significativamente al empujar el trineo en ambas velocidades autoseleccionadas, particularmen te en el músculo GA. WP y RP mostraron una relación inversa en los patrones temporales a nivel neuromuscular. Conclusión / Relevancia clínica: Sugerimos usar el trineo mientras se camina para provocar mejoras de la resistencia y correr para desarrollar el fortalecimiento muscular del tren inferior. El trineo demostró ser una herramienta útil para el entrenamiento neuromuscular mostrando diferentes alteraciones de la musculatura del tren inferior, especialmente de la musculatura extensora.
\end{abstract}

Palabras clave: trineo de sobrecarga, modificaciones neuromusculares, EMG, activación muscular 


\section{Correspondence:}

Martin G. Rosario

School of Physical Therapy, Texas Woman's University, Dallas, Texas, United States of America. mrosario1@twu.edu

Submitted: 31/03/2020

Accepted: 24/06/2020 


\section{INTRODUCTION}

Resistance exercise (RE) is an umbrella term referring to exercise requiring the exertion of force against resistance. $\mathrm{RE}$ is an excellent modality for increasing muscle strength, muscle endurance, power, hypertrophy, and motor performance (Fleck \& Kraemer, 1997; Kraemer \& Ratamess, 2004; Medicine, 2009]. Some studies focus on the biology of RE and muscle fiber adaptations, including the production of a proportional gain in all intracellular elements of all-fiber categories while boosting oxidative phosphorylation with an increase in fiber size. The mitochondrial content of detrained fibers remains constant in both number and form, yet as the volume of the fiber decreases, the mitochondrial volume percent increases. Lastly, heavier RE increases hypertrophy of all fiber types, whereas light RE results in no significant hypertrophy during short-term RE over 8 weeks (Staron et al., 1994, Staron et al., 1989; Staron et al., 1991).

Other researchers emphasize the health benefits of enhancing muscular fitness during RE (Williams et al., 2007); higher levels of muscle strength are linked with significantly lower cardio-metabolic risk factor profiles, lower risk of mortality (FitzGerald et al., 2004), fewer cardiovascular disease events (Gale et al., 2007), lower risk of developing functional limitations (Brill et al., 2000), and nonfatal disease (Jurca et al., 2005).

Inquiries targeting the benefits of long term $\mathrm{RE}$ prove to yield improvements in various areas of health, including body composition (Hunter, McCarthy, Bamman, 2004), blood glucose levels (Castaneda et al. 2002), insulin sensitivity (Brooks et al., 2007), and blood pressure regulation (Collier, Kanaley, Carhart et al., 2009). RE also facilitates muscle strength and mass, which improves bone mass and may assist as a valuable measure to forestall, slow, or even rescind bone mass loss in people with osteoporosis (Kohrt et al., 1985). Additionally, mental health advantages associated with $\mathrm{RE}$ are related to impending and improving depression, anxiety (Cassilhas et al., 2010), and lessening mental fatigue (Puetz, 2006).

Currently, researchers are conducting various types of RE exercises, with many studies growing exponentially. Of particular interest are the expansion of RE exercise selections (i.e., body weight, implements, vibration, and kettlebells) and integrations with other variables, such as sequence, velocity, frequency, and rest intervals (Kraemer et al., 2017). Many of these interests are captured in non-traditional training methods, such as high-intensity power training and CrossFit. Several of these investigations emphasize a more kinematic or kinetic approach rather than neuromuscular recruitment. For instance, Zafeiridis et al. (2005), determined the benefits of pulling a sled in acceleration and speed in young athletes. Alcaraz et al., (2009), focus on the different loads to improve acceleration and velocity while running and pushing a traditional sled in young 
athletes. Other studies direct on the adequate amount of rest to achieve an efficient workout and avoid detrimental effects in muscle performance due to muscle fatigue (Wong et al., 2017).

The current study attempts to understand the neuromuscular timing activation on leg musculature while walking and running pushing a sled. Previous inquiries exploring the impact muscle activity while pushing a traditional sled reveals an increased activation of the gastrocnemius compared to quadriceps and hamstring muscles (Maddigan et al., 2014). Additionally, this same study indicated that more traditional weightlifting maneuvers (back squats) are more effective at engaging back musculature like erector spinae than the sled. Although the aforementioned is valuable, the current study concentrates on the timing for muscle recruitment rather than the amount of muscle activation.

The XPO Trainer is a novel device that provides low rolling resistance at low speeds with an immediate and automatic proportional increase in resistance with increased speed. Rosario et al., (2019) research the kinematic implications of this particular sled, which proved to modify gait variables such as the step/minute, gait speed, the length of stride, and trunk velocity gait variables. Additionally, Mathis et al., (2020) studied the significance of muscle amplitude or amount in muscle activation during walking and pushing the XPO sled compared to only walking and running. The researchers stated the modification occurred primarily in the quadriceps muscle and not gastrocnemius, tibialis anterior, or hamstring while pushing the sled.

Although the previously mentioned is worthwhile, there are several areas we want to investigate when it comes to pushing this specific type of resistance and sled. First, what is the modification in muscle timing recruitment? Several pieces of research focus on the amount or intensity of activation however, the time to reach said activation would be practical to understand. Second, many of the sled studies converge on athletes and athlete's training. However, could it be possible to understand the muscle activation timing and apply tools such as the sled in participants with musculoskeletal and neurological injuries? Finally, since the main focus of the above-mentioned studies in athletes, many of the said inquiries are while sprinting. Could it be possible to utilize this atypical sled resistance at lower speeds such as walking to modify or treat gait deviation?

Based on the above mentioned, the purpose of this study is to examine the impact of using the XPO Trainer sled at low and fast self-selected speeds on muscle activation timing (neuromuscular activation) in young, apparently healthy adults. We hypothesized while pushing the sled, participants will: 1) display evident neuromuscular modifications compared to their counterparts (between running pushing and run, for instance) and 2) indicate a more pronounced neuromuscular adaptation with the extensor musculature, like the 
gastrocnemius, in comparison to flexor musculature, such as the tibialis anterior, due to increased speed and resistance.

\section{Method}

The enlisting process for this inquiry was through word of mouth at the Texas Woman's University (TWU) Dallas Campus and its surrounding communities.

\section{Participants}

Thirty-Six healthy male and female adults (age: $24.6 \pm 3.6$ years, weight: 154.6 \pm 33.1 lbs, height: $66.3 \pm 3.9$ inches) from TWU and its surrounding communities volunteered and contributed to this study (Table 1).

\section{TABLE 1}

Demographic data of all participants.

\begin{tabular}{ll}
\hline \hline Characteristics & Study Participants $\mathrm{n}=36$ \\
\hline Age (years) & $24.6 \pm 3.6$ \\
\hline Gender & Male $=11 ;$ Female $=25$ \\
\hline Height (inches) & $\mathrm{M}=66.3 \pm 3.9$ \\
\hline Weight (pounds) & $154.6 \pm 33.1$ \\
\hline Isometric Muscle Contraction TA & $82.6 \pm 33.0$ \\
\hline Isometric Muscle Contraction GA & $88.7 \pm 40.1$ \\
\hline \hline
\end{tabular}

\section{Procedures}

Those interested in taking part in this study were asked basic wellness questions, such as "Do you have any known heart conditions?" and, "Have you had any injuries to your lower back or lower limbs in the last six months?" to ascertain adequate qualifications to participate in this study. After being deemed as qualifiers, participants were presented with detailed information about the procedures for this study.

Each subject read and signed an informed consent form (Protocol A20091) after being educated on their rights, as well as potential discomforts and risks they may encounter while taking part in this study. After signing the informed consent, participants' information (age, weight, and height) were recorded at the beginning of the session.

For the current study, as inclusion and exclusion criteria to participate, volunteers were required to have the ability to walk without an assistive device, bear standing for at least $30 \mathrm{~min}$, and push a 60-lb sled, as well as have a BMI of $<40$ and a stable cardiorespiratory system, which was assessed by blood pressure and heart rate measurements. To participate in this study, and for the safety of each subject, we required an assessment of the cardiovascular systems 
by measuring blood pressure, heart rate, and oximeter readings to ensure cardiovascular stability after 5 min of sitting (rest period).

EMG Preparation. For the electromyography TRIGNO Avanti surface electrode system (EMG) (Delsys, Inc. Boston, MA), we examined and utilized the position of the electrode on the leg muscles based on Sacco \& Kasman protocols (Criswell, 2011) EMG was engaged in obtaining electrical activity of muscles at $1,000 \mathrm{~Hz}$. Participants were asked to identify their dominant leg. To identify the anterior tibialis, we asked the participant to stand supported with a hand on the chair, standing on the heel (toes in the air) of the dominant extremity during ankle dorsiflexion. The lateral head of the gastrocnemius muscle was assessed by having the partakers rise (ankle plantarflexion) their heel off the floor and push onto their toes. After locating the muscle bellies for the Tibialis Anterior (TA) and lateral Gastrocnemius (GA), the area was cleaned and shaved with a non-electric razor, when applicable. After the targeted area was cleaned and dry, researchers placed electromyography (EMG) (Delsys) surface electrodes on the TA and GA muscle bellies, as seen in Figure 1.

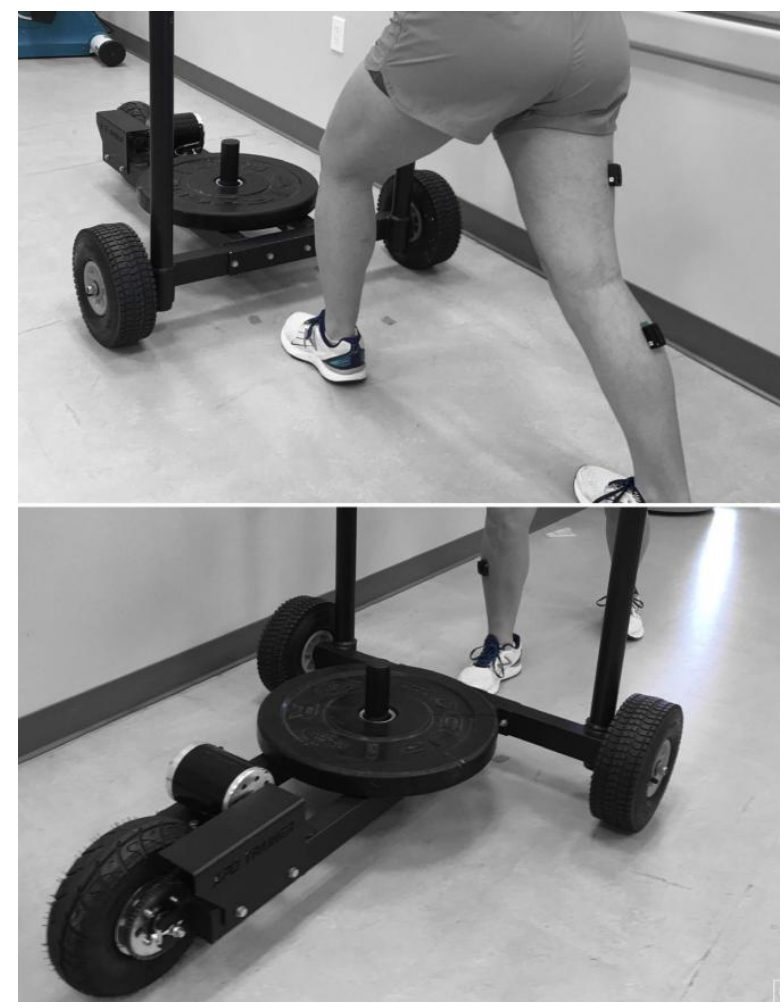

FIGURE 1: Sled XPO Trainer with surface EMG electrode placement on Tibialis Anterior, Gastrocnemius. 
Gait Protocol. Data was collected on hard concrete, even surfaced hallway spanning 80 feet $(24.4 \mathrm{~m})$. Within those 80 feet, a member of the research team measured a 40 -feet $(12.2 \mathrm{~m}$ ) length and marked it by two cones, one placed at the start of the 40 feet, and one placed at the end. Participants were asked to perform 4 tasks at a self-selected speed, walk, run, walk push, and run push. A practice trial for each task was performed to ensure warming up the muscles and that subjects understood the instructions prior to the data collection period. Each task had 3 trials and a minimum of a minute of rest in between tasks to avoid fatigue. Additionally, the order of the tasks was randomized to avoid fatigue as a factor altering the findings in the current study. When working with musculoskeletal injuries and neurological clients, the starting point for treatment is self-selected speed, therefore the current study aims to, in the future, investigate additional populations other than athletes, and presently, to understand the effects of self-select speeds with an established distance, 40 feet.

Then instructions were given to the participants to execute each protocol task by starting at the first cone and stopping after they passed the second cone. All EMG data collection occurred over the measured 40-foot distance for all of the tasks. The two gait tasks without pushing were 1) walking and 2) running, each participant did 3 trials of each task. For the pushing task participants used a sled for walking pushing (WP) and running pushing (RP) (3 trials each). In this study we utilized the XPO sled trainer (Figure 1), this tool provides low rolling resistance at low speeds with an immediate and automatic proportional increment in opposition to an increased rate (www.armoredfitness.com).

Then, each subject practiced pushing the sled prior to performing the three trials for each condition. The order of the tasks was, walk, WP, run, and RP for a total of 12 trials with at least a minute between trials to avoid fatigue. The timer and data collection started at the word "go," and stopped when the back wheel of the sled passed the second cone. This study collected EMG data for the TA and GA during all four conditions, walk, WP, run, and RP.

\section{Data Analysis}

EMG data was captured by EMG works and processed by EMG analysis for both the TA and GA muscles. Investigators distinguished three activation points during the EMG trace for each muscle during all trials. The variable of interest in this endeavor is the time to onset muscle activation (onset). Furthermore, this investigation collected time to maximal peak activation (TP), decay or relaxation of maximal peak activation (decay), and duration of muscle activation (duration). The EMG data collection was an average of 3 consecutive activation points for each muscle during all trials and tasks. For the data analysis, this study utilized SPSS (version 25) with a repeated-measures 
ANOVA to compare the means for all neuromuscular-time variables. This project examined the impact of the sled's resistance on all variables of interest (onset, TP, decay, and duration) by comparing sessions (walk versus WP or run versus RP) and the two muscles' timing within tasks (TA versus GA while WP or RP). To adapt to the repeated measures ANOVA, in this work, we consider a pvalue of $<0.01$ as statistically significant.

\section{RESUlTS}

Table 2 depicts the equivalence of the walk and WP/RP for both the TA and GA muscles. The vast majority of the differences in neuromuscular timing are shown in a modification pattern between comparing walk to RP.

Table 3 demonstrates the neuromuscular timing variables of the running sessions in comparison to WP/RP for both muscles, where the timing outcomes displayed a prolonged time for neuromuscular activation pattern while walking pushing the sled. Moreover, Table 3 illustrates the significant modification of most variables $(\mathrm{p}<0.01)$, particularly during the run and WP comparisons.

Table 4 portrays the equivalence of WP to RP for all neuromuscular variables of interest among the TA and GA muscles. Results showed significant ( $\mathrm{p}<0.01$ ) timing modifications for all variables when comparing WP to RP. Additionally, pushing the sled showed significant $(\mathrm{p}<0.01)$ adjustments in reducing neuromuscular recruitment when walking in comparison to running. 
TABLE 2

Comparisons of EMG variables among tasks. Results of repeated measure ANOVA performed comparing walk and WP/RP. Significance level set at $\mathrm{p} \leq 0.01$.

\begin{tabular}{|c|c|c|c|}
\hline Tibialis Anterior & Means and SD & Means and SD & P Value \\
\hline & \multirow{2}{*}{ Onset Walk: $0.2 \pm 0.2$} & WP:0.2 \pm 0.02 & 0.32 \\
\hline & & $\mathrm{RP}: 0.07 \pm 0.1$ & 0.01 \\
\hline & \multirow{2}{*}{ TP Walk: $0.4 \pm 0.1$} & WP: $0.5 \pm 0.2$ & 0.4 \\
\hline & & $\mathrm{RP}: 0.2 \pm 0.1$ & 0.001 \\
\hline & \multirow{2}{*}{ Decay Walk : $0.3 \pm 0.1$} & WP: $0.4 \pm 0.1$ & 0.05 \\
\hline & & RP: $0.3 \pm 0.1$ & 0.72 \\
\hline & \multirow{2}{*}{ Duration Walk: $0.7 \pm-0.2$} & WP: $1.0 \pm 0.3$ & 0.01 \\
\hline & & $\mathrm{RP}: 0.5 \pm 0.1$ & 0.01 \\
\hline \multirow[t]{9}{*}{ Gastrocnemius } & Means and SD & Means and SD & P Value \\
\hline & \multirow{2}{*}{ Onset Walk: $0.3 \pm 0.2$} & WP:0.5 \pm 0.3 & 0.01 \\
\hline & & $\mathrm{RP}: 0.1 \pm 0.1$ & 0.001 \\
\hline & \multirow{2}{*}{ TP Walk: $0.4 \pm 0.2$} & WP: $0.4 \pm 0.2$ & 1.0 \\
\hline & & $\mathrm{RP}: 0.3 \pm 0.1$ & 0.01 \\
\hline & \multirow{2}{*}{ Decay Walk:0.3 \pm 0.2} & WP:0.3 \pm 0.1 & 0.18 \\
\hline & & $\mathrm{RP}: 0.2 \pm 0.1$ & 0.01 \\
\hline & \multirow{2}{*}{ Duration Walk:0.7 \pm 0.2} & WP: $1.0 \pm 0.2$ & 0.28 \\
\hline & & RP:0.5 \pm 0.1 & 0.001 \\
\hline
\end{tabular}

${ }^{\wedge} W P=$ Walk and Push, RP=Run and Push, S.D.=Standard Deviation

TABLE 3

Comparisons of EMG variables among tasks. Results of repeated measure ANOVA performed comparing run and WP/RP. Significance level set at $\mathrm{p} \leq 0.01$.

\begin{tabular}{|c|c|c|c|}
\hline Tibialis Anterior & Means and SD & Means and SD & P Value \\
\hline & Onset Run:0.07 \pm 0.05 & $\begin{array}{l}\text { WP: } 0.2 \pm 0.02 \\
\text { RP: } 0.07 \pm 0.1\end{array}$ & $\begin{array}{l}\mathbf{0 . 0 1} \\
0.94\end{array}$ \\
\hline & \multirow{2}{*}{ TP Run: $0.2 \pm 0.1$} & WP: $0.5 \pm 0.2$ & 0.001 \\
\hline & & $\mathrm{RP}: 0.2 \pm 0.1$ & 0.27 \\
\hline & \multirow{2}{*}{ Decay Run:0.2 \pm 0.1} & WP: $0.4 \pm 0.1$ & 0.01 \\
\hline & & RP: $0.3 \pm 0.1$ & 0.05 \\
\hline & \multirow{2}{*}{ Duration Run: $0.5 \pm 0.1$} & WP: $1.0 \pm 0.3$ & 0.001 \\
\hline & & RP: $0.5 \pm 0.1$ & 0.19 \\
\hline \multirow[t]{9}{*}{ Gastrocnemius } & Means and SD & Means and SD & P Value \\
\hline & \multirow{2}{*}{ Onset Run:0.1 \pm 0.1} & WP: $0.5 \pm 0.3$ & 0.001 \\
\hline & & $\mathrm{RP}: 0.1 \pm 0.1$ & 0.30 \\
\hline & \multirow{2}{*}{ TP Run:0.3 \pm 0.1} & WP: $0.4 \pm 0.2$ & 0.001 \\
\hline & & $\mathrm{RP}: 0.3 \pm 0.1$ & 0.90 \\
\hline & \multirow{2}{*}{ Decay Run:0.2 \pm 0.1} & WP: $0.3 \pm 0.1$ & 0.01 \\
\hline & & $\mathrm{RP}: 0.2 \pm 0.1$ & 0.15 \\
\hline & \multirow{2}{*}{ Duration Run: $0.5 \pm 0.1$} & WP: $1.0 \pm 0.2$ & 0.001 \\
\hline & & RP: $0.5 \pm 0.1$ & 0.54 \\
\hline
\end{tabular}

${ }^{\wedge} W P=$ Walk and Push, $R P=$ Run and Push, S.D.=Standard Deviation 
TABLE 4

Comparisons of EMG variables among tasks. Results of repeated measure ANOVA performed comparing RP and WP. Significance level set at $\mathrm{p} \leq 0.01$.

\begin{tabular}{llll}
\hline \hline Tibialis Anterior & Means and SD & Means and SD & P Value \\
\hline & Onset RP:0.07 \pm 0.1 & WP:0.2 \pm 0.02 & $\mathbf{0 . 0 1}$ \\
\cline { 2 - 4 } & TP RP:0.2 \pm 0.1 & WP: $0.5 \pm 0.2$ & $\mathbf{0 . 0 0 1}$ \\
\cline { 2 - 4 } & Decay RP:0.3 \pm 0.1 & WP: $0.4 \pm 0.1$ & $\mathbf{0 . 0 1}$ \\
\cline { 2 - 4 } & Duration RP:0.5 \pm 0.1 & WP: $1.0 \pm 0.3$ & $\mathbf{0 . 0 0 1}$ \\
\hline Gastrocnemius & Means and SD & Means and SD & P Value \\
\hline & Onset RP:0.1 \pm 0.1 & WP:0.5 \pm 0.3 & $\mathbf{0 . 0 0 1}$ \\
\cline { 2 - 4 } & TP RP:0.3 \pm 0.1 & WP:0.4 \pm 0.2 & $\mathbf{0 . 0 0 1}$ \\
\cline { 2 - 4 } & Decay RP:0.2 \pm 0.1 & WP:0.3 \pm 0.1 & $\mathbf{0 . 0 1}$ \\
\cline { 2 - 4 } & Duration RP:0.5 \pm 0.1 & WP: $1.0 \pm 0.2$ & $\mathbf{0 . 0 0 1}$ \\
\hline \hline
\end{tabular}

${ }^{\wedge} W P=$ Walk and Push, $R P=$ Run and Push, S.D.=Standard Deviation

Finally, Table 5 delineates the neuromuscular timing when comparing both TA and GA muscles for all of the tasks. When exploring the patterns of timing activation, results show both muscles considerably $(\mathrm{p}<0.01)$ adapted their timing parameters when pushing the sled. The variable that showed the most substantial $(\mathrm{p}<0.01)$ alteration was the decay (prolong activation) of both the GA and TA during the RP task. 
TABLE 5

Comparison of neuromuscular timing between muscles. Results of repeated measure ANOVA performed comparing TA and GA muscles. Significance level set at $\mathrm{p} \leq 0.01$.

\begin{tabular}{llll}
\hline \hline & TA & GA & P value \\
\hline Onset & & & \\
\hline Walk & $0.2 \pm 0.2$ & $0.3 \pm 0.2$ & 0.2 \\
\hline WP & $0.2 \pm 0.02$ & $0.5 \pm 0.3$ & $\mathbf{0 . 0 0 1}$ \\
\hline Run & $0.07 \pm 0.05$ & $0.1 \pm 0.1$ & 0.1 \\
\hline RP & $0.07 \pm 0.1$ & $0.1 \pm 0.1$ & $\mathbf{0 . 0 1}$ \\
\hline Time to Peak & & & \\
\hline Walk & $0.4 \pm 0.1$ & $0.4 \pm 0.2$ & 1.0 \\
\hline WP & $0.5 \pm 0.2$ & $0.4 \pm 0.2$ & 0.1 \\
\hline Run & $0.3 \pm 0.1$ & $0.3 \pm 0.1$ & 0.7 \\
\hline RP & $0.2 \pm 0.1$ & $0.3 \pm .1$ & 0.05 \\
\hline Decay & & & \\
\hline Walk & $0.3 \pm 0.1$ & $0.3 \pm 0.2$ & 0.3 \\
\hline WP & $0.4 \pm 0.1$ & $0.3 \pm 0.1$ & $\mathbf{0 . 0 1}$ \\
\hline Run & $0.2 \pm 0.1$ & $0.2 \pm 0.1$ & 0.6 \\
\hline RP & $0.3 \pm 0.1$ & $0.2 \pm 0.1$ & $\mathbf{0 . 0 0 5}$ \\
\hline Duration & & & \\
\hline Walk & $0.7 \pm 0.2$ & $0.9 \pm 0.3$ & 0.67 \\
\hline WP & $1.0 \pm 0.2$ & $0.7 \pm 0.2$ & $\mathbf{0 . 0 1}$ \\
\hline Run & $0.5 \pm 0.1$ & $0.5 \pm 0.1$ & 0.9 \\
\hline RP & $0.5 \pm 0.1$ & $0.5 \pm 0.1$ & 0.5 \\
\hline \hline
\end{tabular}

${ }^{\wedge} W P=$ Walk and Push, RP=Run and Push, S.D.=Standard Deviation

\section{DisCUSSION}

Previous studies focused on this particular sled and determined the modifications in gait variables (Rosario et al., 2019) and maximal muscle activation of lower limb musculature (Mathis et al., 2020), however the time to reach said muscle activation is yet to be examined. Therefore, the aim of this study was to investigate the influence of pushing a low rolling constantresistance sled at self-selected walking and running speeds on neuromuscular timing for muscle contraction on the Tibialis Anterior and Gastrocnemius muscles of healthy young adults. We intended to discern the impact of the sled on muscle activation to ascertain specific interventions on participants with unique injuries or pathologies, in the future.

We hypothesized that while pushing the sled, participants would display evident neuromuscular adaptations associated with their counterpart tasks (RP versus run, for instance). Additionally, we speculated that the resistance exerted by the pushing muscles, as in the gastrocnemius (extensor musculature), will reveal more evident neuromuscular changes when compared to the tibialis anterior (flexor musculature) due to increased speed and resistance. 
The outcomes of this study verified the evident, noticeable changes in neuromuscular recruitment while pushing the sled. When differentiating between walking while pushing the sled (WP) and running while pushing the sled (RP), neuromuscular timing modifications were observed mainly in the GA muscle, and primarily at a slower speed. Therefore, we accepted our original hypotheses.

Walking vs. WP and RP. Results of this study provide evidence of faster neuromuscular recruitment (shorter time to onset and TP), prominently observed with an increase in speed. These outcomes suggest that pushing the resistance sled at both speeds causes faster neuromuscular recruitment with reduced muscular activation time. The increase in recruitment was, to a great extent, more dominant in the GA when compared to the TA. Previous studies indicate that lower extremity musculature, such as the gastrocnemius and tibialis anterior, are key players in maintaining standing posture (ShumwayCook \& Woollacott, 2007]. Subjects with weak, deficient, or altered neuromuscular activation in GA and TA, such as people living with HIV, have increased sway while standing (Rosario et al., 2020). During gait, the normal functions of these muscles are essential for adequate motor control during gait on any surface (Shumway-Cook \& Woollacott, 2007) or speed. Based on the above, we suggest utilizing the sled as a training tool for participants at a selfselected speed to isolate and concentrate on the GA and TA motor control and motor coordination. Future studies should place emphasis on the effectiveness of an intervention plan using the sled on different lower limb (GA and TA) pathologies or injuries.

Run vs. WP and RP. Data from this study reveals modifications in neuromuscular recruitment while running when compared to pushing the sled, particularly in the WP task. The primary changes were displayed more in the GA (pushing-extensor musculature) than the TA. The pattern that emerged comparing the run task with the WP task was slower recruitment (longer onset and TP) with prolonged activation (increased duration and decay). Research highlights the advantages of RE in skeletal muscles (Kohrt et al., 1985) and average gait speed for adequate functionality and healthy quality of life (Bergland et al., 2017). Weakness of the lower extremities is one of the factors that impact and alter normal gait speed (Tanaka et al., 2019), which in turn can increase the risk of falling (García-Hermoso et al., 2018). In this study, participants self-selected their own safe speed for walking, as well as running. A remarkable result, and benefit of pushing the sled while walking, was the targeted neuromuscular recruitment of the GA muscle. The GA muscle has been studied as a standard for mortality, normal gait, and overall quality of life (Debette et al., 2008), with many researchers proposing to direct strengthening treatment protocols to the extensor musculature to improve and/or enhance 
these aspects (Teixeira-Samela et al., 1999; Osawa et al., 2019; Reynaudet al., 2019; Henderson et al., 2017). Therefore, similar to Guadagnin et al., (2019), we suggest exploring age-related differences and their impact on the GA musculature, though we suggest utilizing this particular sled as an addition to the said future research on gait speed and the GA in distinct lower extremities and neurological pathologies.

$W P$ versus RP. Our study illustrates an inverted association in neuromuscular modifications while walking while pushing the sled and running while pushing the sled; RP showed slower recruitment with a prolonged activation, whereas WP exhibited a faster enlisting with a shorter duration of muscle activation. With this, increased speed in both walking and running while pushing the sled caused faster recruitment (reduced onset and TP) with a shorter activation (reduced duration and decay).

\section{Conclusions}

The findings achieved from this study confirm employing the XPO Trainer sled as a RE tool that furnishes gait and neuromuscular benefits, in addition to the efficacy that traditional walking and running offers. Our study ushers on that the impact of the resistance sled on the investigated muscles are distinct, as each has its own unique increases in muscular activation with different pushing tasks. Modifications that arise from pushing the sled have now been proven to occur regardless of speed, highlighting the suggestion that clinicians can target specific neuromuscular control patterns, potentially allowing for more time to focus on the gait control of each stride to work towards more proportional gait patterns (Shumway-Cook \& Woollacott, 2007). We speculate that the WP tasks will focus on the promotion of endurance associated with slower muscle recruitment and prolonged duration. In contrast, the RP task could potentially target strengthening related to the faster muscular activation recruitment over a shorter period, thus delaying the occurrence of muscle fatigue. Like previous studies focusing on the benefits of resistance training, including improvement in walking speed and stride length in specific populations (Park et al., 2015), we suggest utilizing the sled for similar objectives. Discerning the effects that this resistance sled can have on a variety of components of the muscle responsible for gait and posture is advantageous for clinically relevant applications to distinct folks. Future studies should emphasize on different pathologies and populations, as well as upon recognizing the usefulness of various distances and surfaces.

\section{ACKNOWLEDGMENTS}

I gratefully acknowledge the contributions of Monica Mathis, and Aneesah Hyder to this project. 


\section{REFERENCES}

Alcaraz, P.E., Palao, J.M. \& Elvira, J.L. (2009). Determining the optimal load for resisted sprint training with sled towing. Journal of Strength and Conditioning Research, 23(2):480-485. doi:10.1519/JSC.0b013e318198f92c.

Armored fitness equipment. Retrieved March 15, 2020. https://www.armoredfitness.com

Bergland, A., Jørgensen, L., Emaus, N. \& Strand, B. H. (2017). Mobility as a predictor of all-cause mortality in older men and women: 11.8 year followup in the troms $\varnothing$ study. BMC Health Services Research, 17(1): 22. doi:10.1186/s12913-016-1950-0.

Brill, P.A., Macera, C.A., Davis, D.R., Blair, S.N. \& Gordon, N. (2000). Muscular strength and physical function. Medicine and Science in Sport and Exercise, 32(2):412-6.

Brooks, N., Layne, J.E., Gordon, P.L., Roubenoff, R., Nelson, M.E., CastanedaSceppa, C. (2007). Strength training improves muscle quality and insulin sensitivity in Hispanic older adults with type 2 diabetes. International Journal of Medicine and Science, 4(1):19-27.

Cassilhas, R.C., Antunes, H.K., Tufik, S. \& de Mello, M.T. (2010). Mood, anxiety, and serum IGF-1 in elderly men given 24 weeks of high resistance exercise. Perceptual and Motor Skills, 110(1):265-76.

Castaneda, C., Layne, J.E.; Munoz-Orians, L., Gordon, P.L., Walsmith, J., Foldvari, M., Roubenoff, R., Tucker, K.L. \& Nelson, M.E. (2002). A randomized controlled trial of resistance exercise training to improve glycemic control in older adults with type 2 diabetes. Diabetes Care, 25(12):2335-41.

Collier, S.R., Kanaley, J.A., Carhart Jr, R., Frechette, V., Tobin, M.M., Bennett, N., Luckenbaugh, A.N. \& Fernhall, B. (2009). Cardiac autonomic function and baroreflex changes following 4 weeks of resistance versus aerobic training in individuals with pre-hypertension. Acta Physiologica (Oxf), 195(3):33948.

Criswell, E. (2011). Cram's Introduction to Surface Electromyography, Cram J Electromyography. Second Edition. Sudbury, Massachusetts Boston. Jones and Bartlett Publishers.

Debette, S., Leone, N., Courbon, D., Gariepy, J., Tzourio, C., Dartigues, J., Ritchie, K., Alperovitch, A., Ducimetiere, P., Amouyel, P. \& Zureik, M. (2008). Calf circumference is inversely associated with carotid plaques. Stroke, 39(11), 2958-2965. doi: 10.1161/STROKEAHA.108.520106

FitzGerald, S.J.B.C., Kampert, J.B., Morrow Jr, J.R., Jackson, A.W. \& Blair, S.N. (2004). Muscular fitness and all-cause mortality: a prospective study. Journal of Physical Activity and Health, 1:7-18.

Fleck, S.J., Kraemer, W.J. (1997). Designing resistance training programs. 2nd ed. Champaign (IL): Human Kinetics. 
Gale, C.R., Martyn, C.N., Cooper, C. \& Sayer, A.A. (2007). Grip strength, body composition, and mortality. International Journal of Epidemiology, 36(1):228-35.

García-Hermoso, A., Cavero-Redondo, I., Ramírez-Vélez, R., Ruiz, J. R., Ortega, F. B., Lee, D. \& Martínez-Vizcaíno, V. (2018). Muscular strength as a predictor of all-cause mortality in an apparently healthy population: A systematic review and meta-analysis of data from approximately 2 million men and women. Archives of Physical Medicine and Rehabilitation, 99: 2100-2113. doi: 10.1016/j.apmr.2018.01.008.

Guadagnin, E.C., Barbieri, F.A., Simieli, L. \& Carpes, F.P. (2019). Is muscular and functional performance related to gait symmetry in older adults? A systematic review. Archives of Gerontology and Geriatrics, 84: 1-6. doi: 10.1016/j.archger.2019.103899.

Henderson, R.M., Leng, X.I., Chmelo, E.A., Brinkley, T.E., Lyles, M.F., Marsh, A.P. \& Nicklas, B.J. (2017). Gait speed response to aerobic versus resistance exercise training in older adults. Aging Clinical and Experimental Research, 29(5), 969-976.

Hunter, G.R., McCarthy, J.P. \& Bamman, M.M. (2004). Effects of resistance training on older adults. Sports Medicine, 34(5):329-48.

Jurca, R., LaMonte, M.J., Barlow, C.E., Kampert, J.B., Church, T.S. \& Blair, S.N. (2005). Association of muscular strength with incidence of metabolic syndrome in men. Medicine and Science in Sport and Exercise, 37(11):184955.

Kohrt, W.M., Bloomfield, S.A., Little, K.D., Nelson, M.E. \& Yingling, V.R. (2004). American College of Sports Medicine. Position Stand: physical activity and bone health. Medicine and Science in Sport and Exercise, 36(11): 1985-96.

Kraemer, W.J. \& Ratamess, N.A. (2004). Fundamentals of resistance training: progression and exercise prescription. Medicine and Science in Sport and Exercise, 36:674-8.

Kraemer, W.J., Ratamess, N.A., Flanagan, S.D., Shurley, J.P., Todd, J.S. \& Todd, T.C. (2017) Understanding the science of resistance training: An evolutionary perspective. Sports Medicine, 47(12): 2415-2435.

Maddigan, M.E., Button, D.C. \& Behm, D.G. (2014). Lower-limb and trunk muscle activation with back squats and weighted sled apparatus. Journal of Strength and Conditioning Research, 28(12):3346-3353. doi:10.1519/JSC.0000000000000697.

Mathis, M., Timon, S., Taylor, H. \& Rosario, M. G. (2020). Muscle Activation Response Using a Constant Resistance Sled Trainer Within Different Tasks. The FASEB Journal, 34(S1): 1-1. 
Medicine ACoS. (2009). Position stand: progression models in resistance training for healthy adults. Medicine and Science in Sport and Exercise, 41:687-708.

Osawa, Y., Shaffer, N.C., Shardell, M.D., Studenski, S.A. \& Ferrucci, L. (2019). Changes in knee extension peak torque and body composition and their relationship with change in gain speed. Journal of Cachexia, Sarcopania and Muscle, 10(5): 1000-1008. doi: 10.1002/jcsm.12458.

Park, B.S., Kim, M.Y., Lee, L.K., Yang, S.M., Lee, W.D., Noh, J.W. \& Kim, J. (2015). The effects of a progressive resistance training program on walking ability in patients after stroke: a pilot study. Journal of Physical Therapy Science, 27(9): 2837-2840. doi: 10.1589/jpts.27.2837.

Puetz, T.W. (2006). Physical activity and feelings of energy and fatigue: epidemiological evidence. Sports Medicine, 36(9):767-80.

Reynaud, V., Morel, C., Givron, P., Clavelou, P., Cornut-Chauvinc, C., Pereira, B., Taithe, F. \& Coudeyre, E. (2019). Walking speed is correlated with the isometric muscular strength of the knee in patients with chariot-marietooth type 1a. American Journal of Physical Medicine and Rehabilitation, 98(5): 422-425. doi: 10.1097/PHM.0000000000001084.

Rosario, M.G., Hyder, A., Mathis, M., Timon, S., Taylor, H. \& Swank, C. (2019). Pushing A Sled: Assessing Its Impact On Gait Temporospatial Parameters In Young Healthy Adults. Medicine and Science in Sports and Exercise, 51(6): 960. doi: 10.1249/01.mss.0000563381.65694.d3.

Rosario, M.G., Marshall, J., Herkert, A., Binoy, B., Windham, H., \& Orozco, E. (2020). Lower Limb Neuromuscular Modification and Standing Postural Control Alteration in Apparent Asymptomatic People Living with HIV. Journal of Rehabilitation Practices and Research, 1(1):102. doi:10.33790/jrpr1100102.

Schoenfeld, B.J., Grgic, J., Haun, C., Itagaki, T. \& Helms, E.R. (2019) Calculating Set-Volume for the Limb Muscles with the Performance of Multi-Joint Exercises: Implications for Resistance Training Prescription. Sports (Basel), 7(7):177. doi: 10.3390/sports7070177.

Shumway-Cook, A \& Woollacott, M. H. (2007). Motor control: Translating research into clinical practice. Philadelphia: Lippincott Williams \& Wilkins. APA (6th ed.)

Staron, R. S., Karapondo, D. L., Kraemer, W. J., Fry, A. C., Gordon, S. E., Falkel, J. E., Hagerman, F. C., \& Hikida, R. S. (1994). Skeletal muscle adaptations during early phase of heavy-resistance training in men and women. Journal of applied physiology, 76(3): 1247-1255. https://doi.org/10.1152/jappl.1994.76.3.1247.

Staron, R. S., Leonardi, M. J., Karapondo, D. L., Malicky, E. S., Falkel, J. E., Hagerman, F. C., \& Hikida, R. S. (1991). Strength and skeletal muscle 
adaptations in heavy-resistance-trained women after detraining and retraining. Journal of Applied Physiology, 70(2), 631-640. https://doi.org/10.1152/jappl.1991.70.2.631

Staron, R.S., Malicky, E.S., Leonardi, M.J., Falkel, J.E., Hagerman, F.C. \& Dudley, G.A. (1989). Muscle hypertrophy and fast fiber type conversions in heavy resistance-trained women. European Journal of Applied Physiology, 60:71-9.

Tanaka, M., Ikezoe, T., Ichihashi, N., Tabara, Y., Nakayama, T., Takahashi, Y., Matsuda, F. \& Tsuboyama, T. (2019). Relationship of low muscle mass and obesity with physical function in community dwelling older adults. Results from the Nagahama study. Archives of Gerontology and Geriatrics. doi: 10.1016/j.archger.2019.103987.

Teixeira-Samela, L.F., Olney, S.J., Nadeau, S. \& Brouwer, B. (1999). Muscle strengthening and physical conditioning to reduce impairment and disability in chronic stroke survivors. Archive of Physical Medicine and Rehabilitation, 80(10), 1211-1218. doi: 10.1016/s0003-9993(99)90018-7.

Williams, M.A., Haskell, W.L., Ades, P.A., Amsterdam, E.A., Bittner, V., Franklin, B.A., Gulanick, M., Laing, S.T. \& Stewart, K.J. (2007). Resistance exercise in individuals with and without cardiovascular disease: 2007 update: a scientific statement from the American Heart Association Council on Clinical Cardiology and Council on Nutrition, Physical Activity, and Metabolism. Circulation, 116(5): 572-84.

Wong, M.A., Dobbs, I.J., Watkins, C.M., Barillas, S.R., Lin, A., Archer, D.C., Lockie, R.G., Coburn, J.W. \& Brown L.E. (2017). Sled Towing Acutely Decreases Acceleration Sprint Time. Journal of Strength and Conditioning Research 31(11):3046-3051. doi:10.1519/JSC.0000000000002123. 